\title{
Probing polarization and dielectric function of molecules with higher order harmonics in scattering-near-field scanning optical microscopy
}

\author{
Maxim P. Nikiforov, ${ }^{1, a)}$ Susanne C. Kehr, ${ }^{2,3}$ Tae-Hong Park ${ }^{4}$ Peter Milde, ${ }^{2}$ Ulrich Zerweck, ${ }^{2}$ \\ Christian Loppacher, ${ }^{5}$ Lukas M. Eng, ${ }^{2}$ Michael J. Therien, ${ }^{6}$ Nader Engheta, ${ }^{7}$ and \\ Dawn Bonnell \\ ${ }^{1}$ The Center for Nanophase Materials Sciences, Oak Ridge National Laboratory, Oak Ridge, Tennessee \\ 37831, USA \\ ${ }_{2}^{2}$ Institute of Applied Photophysics, Technische Universität Dresden, Dresden, 01069, Germany \\ ${ }^{3}$ Department of Physics, University of California, Berkeley, California 94720, USA \\ ${ }_{5}^{4}$ Department of Chemistry, University of Pennsylvania, Philadelphia, Pennsylvania 19104, USA \\ ${ }^{5}$ IM2NP-UMR CNRS 6242 and Aix-Marseille University, F-13397 Marseille Cedex 20, France \\ ${ }^{6}$ Department of Chemistry, Duke University, Durham, North Carolina 27708, USA \\ ${ }^{7}$ Department of Electrical and Systems Engineering, University of Pennsylvania, Philadelphia, Pennsylvania \\ 19104, USA \\ ${ }^{8}$ Department of Materials Science and Engineering, University of Pennsylvania, Philadelphia, Pennsylvania \\ 19104, USA
}

(Received 4 February 2009; accepted 19 September 2009; published online 3 December 2009)

\begin{abstract}
The idealized system of an atomically flat metallic surface [highly oriented pyrolytic graphite (HOPG)] and an organic monolayer (porphyrin) was used to determine whether the dielectric function and associated properties of thin films can be accessed with scanning-near-field scanning optical microscopy (s-NSOM). Here, we demonstrate the use of harmonics up to fourth order and the polarization dependence of incident light to probe dielectric properties on idealized samples of monolayers of organic molecules on atomically smooth substrates. An analytical treatment of light/ sample interaction using the s-NSOM tip was developed in order to quantify the dielectric properties. The theoretical analysis and numerical modeling, as well as experimental data, demonstrate that higher order harmonic scattering can be used to extract the dielectric properties of materials with tens of nanometer spatial resolution. To date, the third harmonic provides the best lateral resolution $(\sim 50 \mathrm{~nm})$ and dielectric constant contrast for a porphyrin film on HOPG.
\end{abstract}

(C) 2009 American Institute of Physics. [doi:10.1063/1.3245392]

\section{INTRODUCTION}

In recent years a family of scanning probe microscopy (SPM) techniques based on applying multiple modulations to samples and tips, and accessing higher order harmonics has been evolving. ${ }^{1,2}$ Scanning surface potential microscopy (SSPM) or Kelvin force microscopy (KFM) is the first and most basic of this approach. This family now includes scanning impedance microscopy, nanoimpedance spectroscopy, scanning capacitance microscopy, etc. ${ }^{3-6}$ This approach can also be extended to optical SPM techniques ranging from UV to IR light ${ }^{7}$ with the potential to access dielectric functions at surfaces. Various techniques have been developed for high-spatial-resolution probes of light interactions with surfaces, including photon assisted scanning tunneling microscopy, surface enhanced Raman spectroscopy, near-field scanning optical microscopy (NSOM), etc. (Ref. 8 and references therein). Of the scanning optical microscopies, so-called scattering-near-field optical microscopy (s-NSOM) offers the best opportunity for high-spatial resolution. ${ }^{9}$ A sharp probe tip is positioned near a surface and illuminated with optical radiation. The field is enhanced at the tip that acts as an optical antenna. Zenhausern et al., ${ }^{10}$ then Hillenbrand and co-wokers, ${ }^{9,11-14}$ modulated the tip-sample distance in the

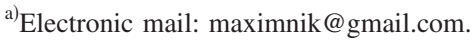

enhanced field, detecting the re-irradiated light to distinguish variations in materials properties near the tip. More recently, contrast due to charge carriers in semiconductors, ${ }^{15}$ and differences between metallic, semiconducting, and insulating compounds ${ }^{11}$ has been demonstrated, by monitoring the second and third harmonics of the scattered signals. In 2008 Wollny et $a l .{ }^{16}$ characterized lipid bilayer stacks on mica with scanning near-field infrared microscopy (SNIM) (a technique very similar to s-NSOM). This group found that SNIM contrast appears on the samples with lipid bilayer stacks thicker than three bilayers. ${ }^{16}$

Relevant dielectric functions are an inherent component of the response of a tip-surface junction in this configuration. For the case of a thin film of organic molecules on a surface, local dielectric properties should, in principle, be accessible in the higher harmonics, provided the volume fraction of interaction is large enough. Here we demonstrate the use of harmonics up to fourth order and polarization dependence of incident light to probe the dielectric properties on idealized samples of monolayers of organic molecules on atomically smooth substrates. Because of the relevance to processes as diverse as photosynthesis and molecular electronics, porphyrin compounds are used as a model molecule. We develop an analytical treatment of light interaction at the tip as a framework within which to consider the potential to quantify dielectric properties. 


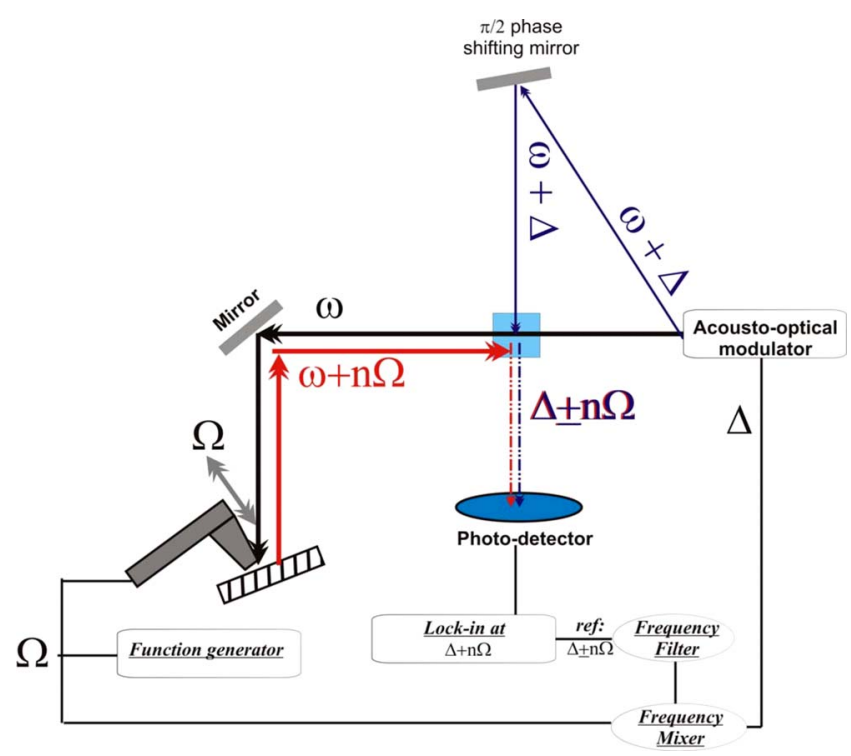

FIG. 1. (Color online) Schematics of the frequency modulated s-NSOM instrument, with the heterodyne detection of the scattered light.

\section{EXPERIMENTAL PROCEDURES}

A Pt-Ir coated Si tip of an atomic force microscope (AFM) was used as an AFM and a s-NSOM probe. Simultaneous imaging of sample topography, amplitude, and phase was performed in a true noncontact AFM regime, using the frequency shift $\Delta f$ to control topography. ${ }^{17}$ The intensity of light scattered from the tip-surface junction was measured using a home-built s-NSOM. ${ }^{7,18,19}$ A schematic of the s-NSOM is presented in Fig. 1. The amplitude and phase of the backscattered light were detected using a heterodyne interferometer under ambient conditions similar to that used by Keilmann and co-workers. ${ }^{20,21} \mathrm{~A}$ focused laser beam with $\lambda$ $=632.8 \mathrm{~nm}$ (He-Ne laser) was sourced to an acousto-optical modulator (AOM) (see the right side of Fig. 1). The AOM splits the beam into two components (primary and modulated). The primary beam (black arrow in Fig. 1) has the same frequency $(\omega)$ as the incoming beam but slightly smaller amplitude. The modulated beam (blue arrow in Fig. $1)$, a mixture of two frequencies $\omega+\Delta(\Delta=70 \mathrm{MHz}$ in this experiment), is used to implement the heterodyne detection scheme. After passing through the AOM, the primary beam reflects off the mirror and illuminates the tip-surface junction. In order to vary the polarization of the incoming light, a polarizer was introduced behind the AOM changing the polarization of both the primary and modulated beams. The path of the scattered light is indicated with red arrows in Fig. 1 . Note that the scattered light is modulated with the harmonics of the tip oscillation frequencies $(\omega+n \Omega)$ due to tip oscillations above the surface during nc-AFM operation. In the beam splitter (light blue square) the scattered beam mixes light scattered from the tip-surface junction with the modulated beam. The resulting beam (marked as dotted red/blue lines in Fig. 1) is modulated with frequencies $(\Delta+n \Omega)$. A fast photodetector with a rise time $\sim 1 \mathrm{~ns}$ was used to convert $(\Delta+n \Omega)$ beam intensity into an electrical signal.

Note that effectively $(\Delta+n \Omega)$ is superimposed on the small topographic frequency shift $\Delta f$ in our frequency modu- lation (FM) setup, hence, feeding the demodulation electronics (lock-in) with the signal $\Delta f+\Delta+n \Omega$ with $\Delta f$ $\sim[0, \ldots, 20 \mathrm{~Hz}], \Delta \sim 70 \mathrm{MHz}$, and $\Omega=150 \mathrm{kHz}$. FMdemodulation s-NSOM is advantageous in order to maintain a constant optical scattering cross section for all $n$ at any position $(x, y, z)$. The lock-in amplifier extracts the amplitude and phase of the scattered signal at frequencies $n \Omega$. The lateral map of the optical amplitude, as well as topography, was obtained by sample movement in the $x-y$ plane. Harmonic signals were detected sequentially and could be associated with topographic position to within $50 \mathrm{~nm}$. The spatial resolution in the optics is limited by the tip radius of curvature, $\approx 5 \mathrm{~nm}$, and the size of the gap between the tip apex and the sample, which changes from 1 to $37 \mathrm{~nm}$ during the tip oscillation. Note that the illuminated region is much larger than $50 \mathrm{~nm}$ (far field). The scattered signal contains a farfield, as well as a near-field contribution, but only the latter is modulated. Detecting scattered light at frequencies $n \Omega$ (a larger harmonic number corresponds to better lateral resolution) minimizes the "background" scattering that is barely modulated. ${ }^{22}$ The tip oscillation frequency was about 150 $\mathrm{kHz}$ and s-NSOM images were obtained at the first, second, third, and fourth harmonics of the tip oscillations $(150,300$, 450 , and $600 \mathrm{kHz}$, correspondingly).

In order to generate s-NSOM maps (Fig. 2) the following procedure was used. The average intensity of scattered light on graphite is calculated $\left(I_{\mathrm{HOPG}}\right)$; the contrast was determined as s-NSOM contrast in Fig. 2 is equal to $[I(x, y)$ $\left.-I_{\mathrm{HOPG}}\right] / I_{\mathrm{HOPG}}$, where $I(x, y)$ is the measured intensity of scattered light as a function of position.

The synthesis of the 5,15-bis[2',6'-bis(3,3-dimethyl1-butyloxy)phenyl] porphyrin complex has been described elsewhere. ${ }^{23}$ For the present experiments, the porphyrin compound was purified by several recrystallizations from hot hexane prior to vacuum deposition. The structure is illustrated in Fig. 3 (for details see supporting information in Ref. $24)$. Note that the steric interaction between the porphyrin $\beta$-hydrogen atoms and the 5- and 15-phenyl ring orthoalkoxy substituents is substantial, and severely restricts the extent of aryl ring vibrational motion as well as the range of accessible torsional angles between the aromatic phenyl ring and porphyrin plane at ambient temperature. Congruent with this fact, x-ray crystallographic studies show that the observed torsional angle between the 5- and 15-aryl rings and the porphyrin plane is $\sim 70^{\circ},{ }^{24}$ and that the phenyl 2', 6'-bis-3,3-dimethyl-1-butyloxy substituents lie approximately orthogonal to the central porphyrin core.

Vacuum deposition of porphyrin molecules was accomplished by heating the solid in a crucible in UHV to a temperature between 130 and $150{ }^{\circ} \mathrm{C}$. The deposition rate in these experiments was $\sim 0.03$ monolayer $/ \mathrm{s}$. A nominal thickness of about 1 monolayer of porphyrin was deposited on highly oriented pyrolytic graphite (HOPG). However, AFM studies subsequently demonstrated that only a submonolayer $(\sim 60 \%)$ coverage was realized.

\section{RESULTS AND DISCUSSION}

Submonolayer deposition of this porphyrin on HOPG results in the formation of islands in which the molecules 

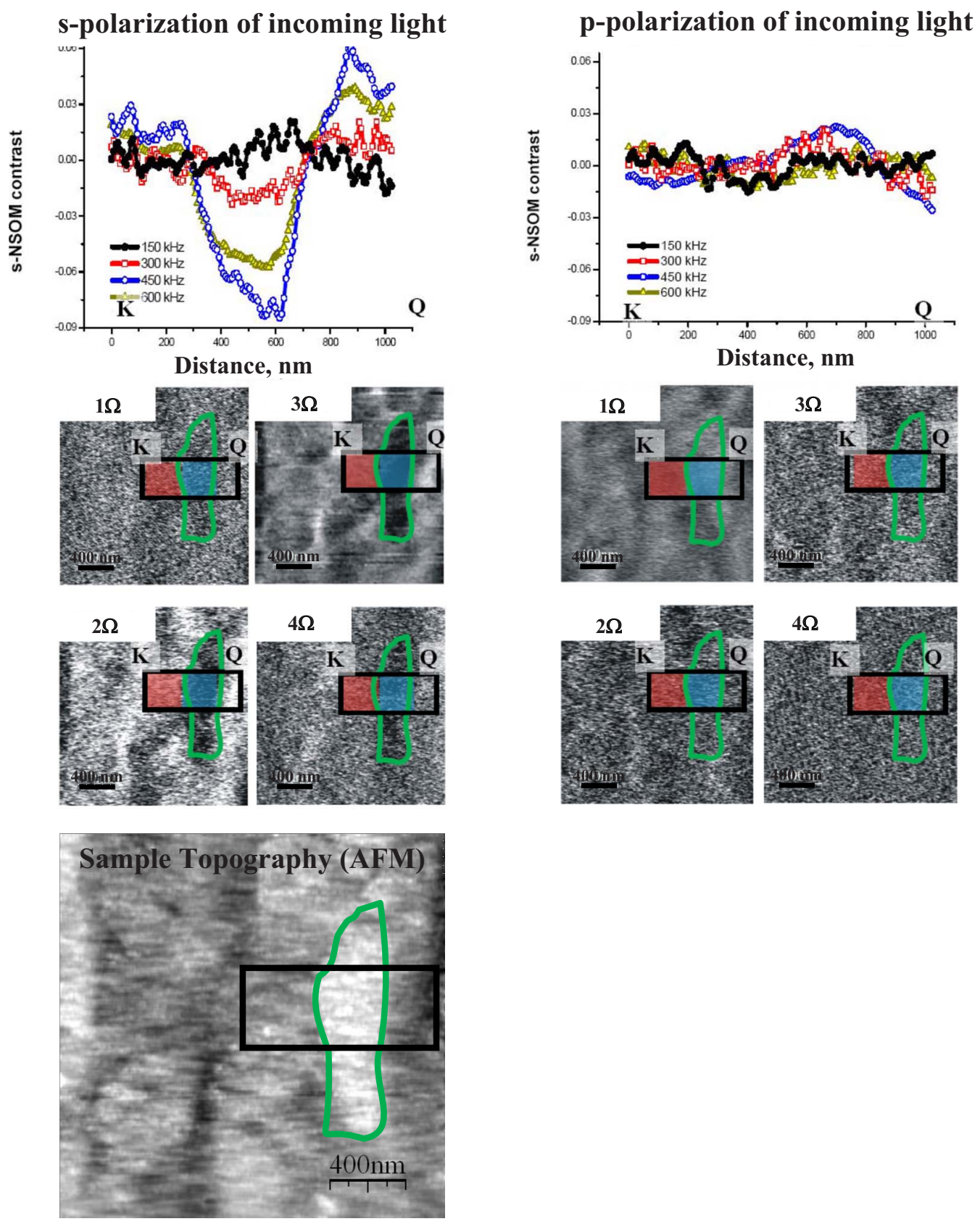

FIG. 2. (Color online) Intensity of the $s$-polarized light (left) and $p$-polarized light (right) scattered from the HOPG/porphyrin-tip junction. The images displayed were acquired, respectively, at $150,300,450$, and $600 \mathrm{kHz}$ (image size $=2 \times 2 \mu \mathrm{m}^{2}$ ). The line cross sections (left and right top plots) were taken as the average of the areas outlined by black boxes in the s-NSOM images. The porphyrin island on HOPG is outlined in green in these images. The sample topography is presented at the bottom of the figure.

adopt two different orientations. In one case the porphyrin ring is oriented parallel to the substrate (phase 2), while in the other it is perpendicular to the surface (phase 1). The different orientations can be distinguished by the height, as shown in Fig. 3. Here the porphyrin islands with the rings parallel to the substrate and a height of $1.5 \mathrm{~nm}$ are examined. The molecular structure of the monolayer is determined from high resolution non-contact AFM, and the locations of the porphyrin phenyl rings with respect to the substrate plane are shown schematically. Note that the aryl substituents stand out of the image plane.

Then, the topographic structure and dependence of the s-NSOM signal on the polarization of the incoming light at multiple harmonics were measured; only s-NSOM reflectance signals are shown in Fig. 2. The porphyrin island is encircled in green and is $1.5 \mathrm{~nm}$ in height, confirming that the molecules align with the porphyrin ring parallel to the HOPG surface. ${ }^{24}$ s-NSOM images were obtained for the four lowest harmonics of the scattered light. The response functions and therefore the properties of the graphite surface and the porphyrin/graphite structure are compared. The porphyrin film does not scatter light as well as HOPG and appears dark in the images. In order to quantify the properties, line profiles across areas with and without the porphyrin monolayer are shown in Fig. 2. The area in red corresponds to the substrate (HOPG), and that in blue corresponds to the por- 

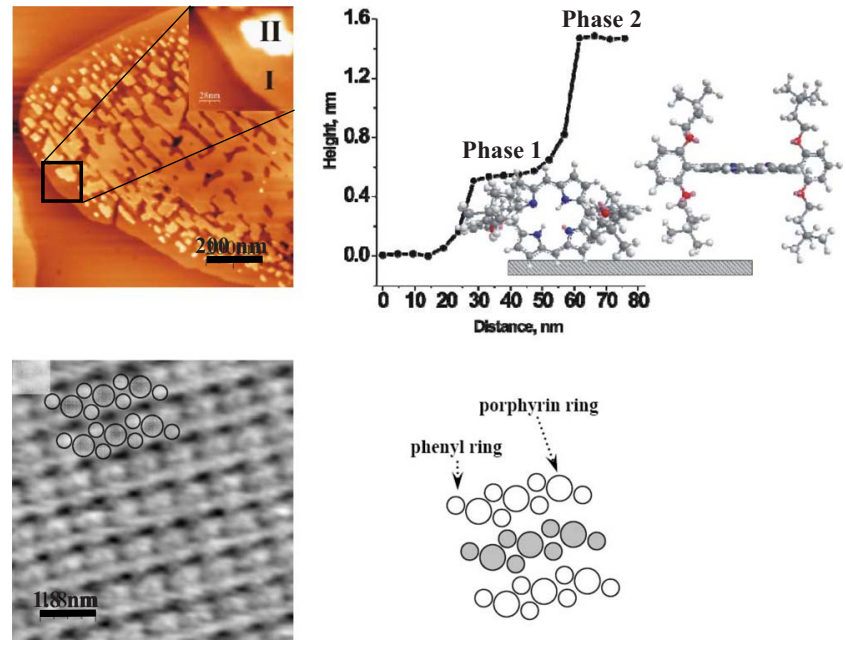

FIG. 3. (Color online) Topography of the porphyrin film on a HOPG substrate (top left image), obtained with low $T$ nc-AFM. Step I corresponds to the molecule where the porphyrin ring is perpendicular to the sample surface (phase 1) while step II corresponds to the molecule with the porphyrin ring parallel to the sample surface (phase 2). The top right image displays a representative height profile of the porphyrin island, showing that step I has an $\sim 0.6 \mathrm{~nm}$ height, when step II has an $\sim 1.5 \mathrm{~nm}$ height. The molecular ordering within step II was measured (bottom left image) and is highlighted in the bottom right image.

phyrin monolayer, indicating again the submonolayer coverage. Clearly when the incident light is polarized perpendicular to the surface ( $p$-polarization) there is almost no difference between the scattered light amplitude at all frequencies. In contrast, when the incident beam is polarized parallel to the surface ( $s$-polarization) the difference in contrast is obvious. Note that the first harmonic signal contains a significant contribution from far-field scattering so the differences are obscured. Nevertheless, the maximum contrast was observed for the third harmonic of the scattered $s$-polarized light.

Interpreting these data requires a quantitative understanding of the light scattering from the tip-surface junction. The simplest description for this light-media interaction is the model of geometrical optics regarding a light beam incident on a planar interface between two media. Depending on the incident angle and media refractive indices, the light will either be reflected by the interface, or transmitted through it with a slight change in direction, or refracted. There are at least two causes of light scattering: roughness of the interface and particles located in the proximity of the surface. In our experiment, freshly cleaved HOPG was used and porphyrin monolayers were grown in UHV yielding surfaces with very small roughness. Although, we observe a submonolayer coverage with step heights of $\sim 0.6$ (phase 1 ) to $\sim 0.9 \mathrm{~nm}$ (phase 2-phase 1), the near-field optical contrast is well defined inside a continuous porphyrin phase revealing a uniform (darker) contrast across the whole porphyrin patch. Hence, scattering from such surface steps is negligible and the contrast stems directly from the tip-porphyrin/HOPG interaction zone. Thus, the theoretical analysis here considers only light scattering due to a particle located near the airHOPG interface.

Various models exist to describe light scattering from a
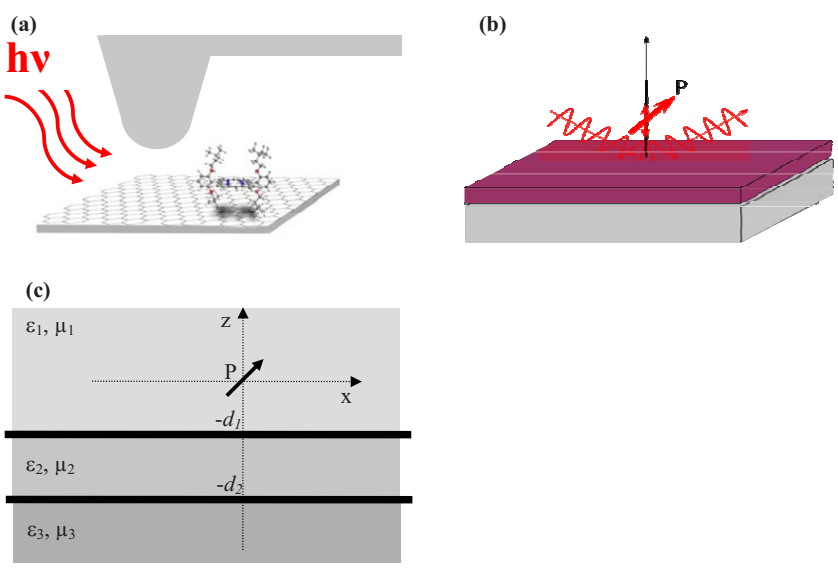

FIG. 4. (Color online) (a) Schematic of the s-NSOM experiment in which the tip is illuminated by red light from the side. (b) Schematic that describes the data analysis model, which treats the probe tip within the context of a point-dipole approximation, and incoming light as a plane wave. (c) Diagrammatic representation of the physical model used in computations of scattered light intensity as a function of tip-substrate distance.

particle located near an interface: the electric dipole model, ${ }^{25,26}$ the electric multipole model, ${ }^{27}$ the electrostatic model, ${ }^{28}$ and the passive probe model. ${ }^{29-31}$ These descriptions fail when the distance between the particle and the surface is smaller than $10 \mathrm{~nm},{ }^{28}$ because it is implicitly assumed that the interaction at the junction does not alter the polarization of scattered light. During our dynamic nc-AFM experiments the gap between the tip apex and the sample changes from 1 to $37 \mathrm{~nm}$; therefore, the simplistic models fail during $25 \%$ of the oscillation cycle. In order to overcome this limitation, we calculate the intensity of scattered light that accounts for the nonlinear enhancement of the electric field (when the tip-surface distance is extremely small) as a function of the time-dependent variation of the tip-sample distance, and apply this analysis to a sample consisting of a substrate and a thin film.

The scattering configuration is illustrated in Fig. 4(a). The interaction of light with a metal tip results in the formation of an induced dipole in the tip, which we treat within the context of a point-dipole approximation, Fig. 4(b). Also, for simplicity, the incoming light is assumed to be a plane wave, which is justified within the focal regime, since the illuminated spot measures $>1 \mu \mathrm{m}$ in diameter compared to an $\sim 100 \mathrm{~nm}$ tip size. Scattered light intensities were calculated for both $s$-polarized (TE) (electric field of incoming light oscillates perpendicular to the plane of incidence) and $p$-polarized (TM) (electric field of incoming light oscillates in the plane of incidence) incoming light. The substrate was considered to be an infinite half space with the properties of graphite having a $1.5 \mathrm{~nm}$ layer with variable dielectric properties, Fig. 4(c). In fact, the goal of these calculations is to determine these dielectric properties. In the experiment, the tip oscillates in proximity to the surface, resulting in a dynamic tip-surface distance, which is modeled in our calculations by varying the distance between the dipole and surface using a sinusoidal function with frequency equal to the frequency of the first tip resonance. Equations derived in the 

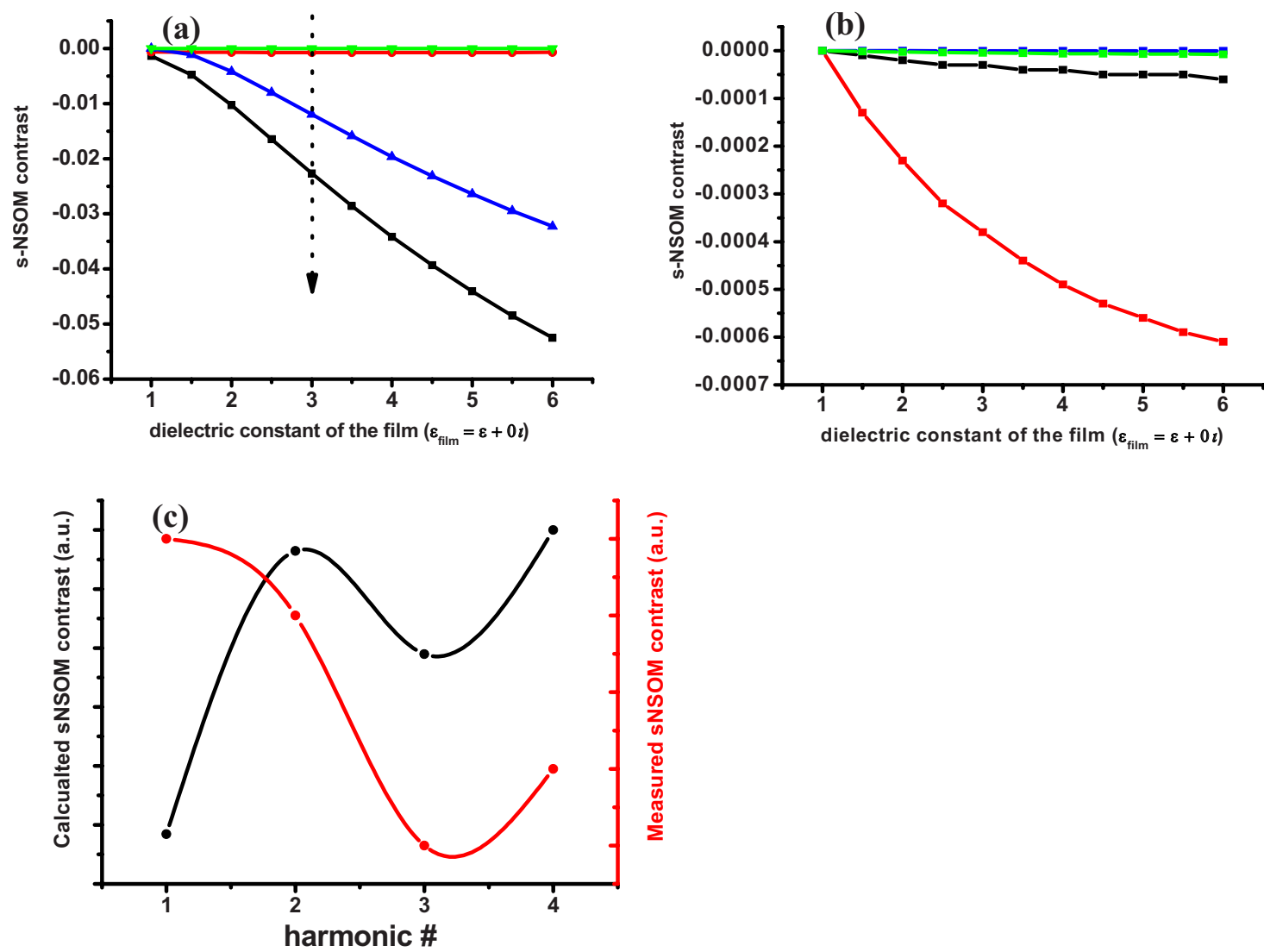

FIG. 5. (Color online) Dependence of the difference between the scattering intensity from the HOPG and HOPG/porphyrin surfaces, normalized by the scattering intensity of the HOPG (ratio proportional to the experimentally measured signal in the s-NSOM experiment) as a function of the film dielectric constant for (a) incoming $s$-polarized light and (b) incoming $p$-polarized light. The results obtained for the first harmonic are presented in black, for the second in red, for the third in blue, and for the fourth in green. (c) The comparison of the s-NSOM contrast calculated (black) and measured (red) for different harmonics between the HOPG and the porphyrin film on the HOPG for a film dielectric constant equal to 3 .

Appendix were used to numerically model the scattered light as a function of the dielectric properties of the substrate and molecular layer.

The difference between the scattered intensity from HOPG and from HOPG/porphyrin normalized by HOPG scattering intensity $\left[\left(I_{\mathrm{HOPG} / \text { porphyrin }}-I_{\mathrm{HOPG}}\right) / I_{\mathrm{HOPG}}\right]$ is directly proportional to the signal measured in our experiment using s-NSOM. In our model, this ratio was calculated using a complex dielectric function for graphite ${ }^{32}$ and a set of real constants for the film. Figures 5(a) and 5(b) compare the s-NSOM contrasts for $s$ - and $p$-polarized light. Note first that the magnitude of the contrast from $p$-polarized scattering is a factor of 100 smaller than that for the $s$-polarized scattering, consistent with the lack of contrast in our experiments [Fig. 2(b)]. It needs to be noted that in our experiment the intensity of the scattered light was larger when the sample was illuminated with $p$-polarized light, than when it was illuminated with the $s$-polarized one. However, the contrast between the HOPG/porphyrin and HOPG (calculated as $\left.\left[\left(I_{\mathrm{HOPG} / \text { porphyrin }}-I_{\mathrm{HOPG}}\right) / I_{\mathrm{HOPG}}\right]\right)$ was weaker when the sample was illuminated with $p$-polarized light, than when it was illuminated with the $s$-polarized one. For the $s$-polarized light, the first harmonic should exhibit the highest contrast, but as noted above, the experimental signal contains a large far-field component that complicates the contrast interpretation. The third harmonic exhibits a second contrast minimum (note that the contrast is negative), consistent with the data in
Fig. 2(a). The calculated contrast in the fourth harmonic is lower than that observed experimentally. Figure 5(c) qualitatively compares the theoretical and experimental contrasts for Fig. 2, the properties expected for the film. With the exception of the experimental artifact in the first harmonic, the agreement is good. With a known dielectric constant for the substrate and calibration of the baseline scattering signal, this procedure can be used to determine the local dielectric constant of the molecular layer.

In 2000 Knoll and F. Keilmann ${ }^{15}$ found that the intensity of scattered light decreases monotonically as a function of harmonic number when a metalized tip is in close proximity of Si or Au surface. This seems to contradict the experimental results presented in this paper. However, this is not the case because in our work the contrast instead of absolute values of scattered s-NSOM signal between two materials is calculated and measured. When the intensity of each harmonic decreases monotonically, the ratio of the harmonics determining the contrast can go through maximum depending on the functional dependence of the monotonic decrease for each harmonic. However, we have to acknowledge that we do not fully understand the underlying reasons for the change in s-NSOM contrast as a function of harmonic number in our case.

Another unusual observation is the increase of the absolute value of the contrast with increasing dielectric value of the film [Figs. 5(a) and 5(b)], i.e., the islands on HOPG 

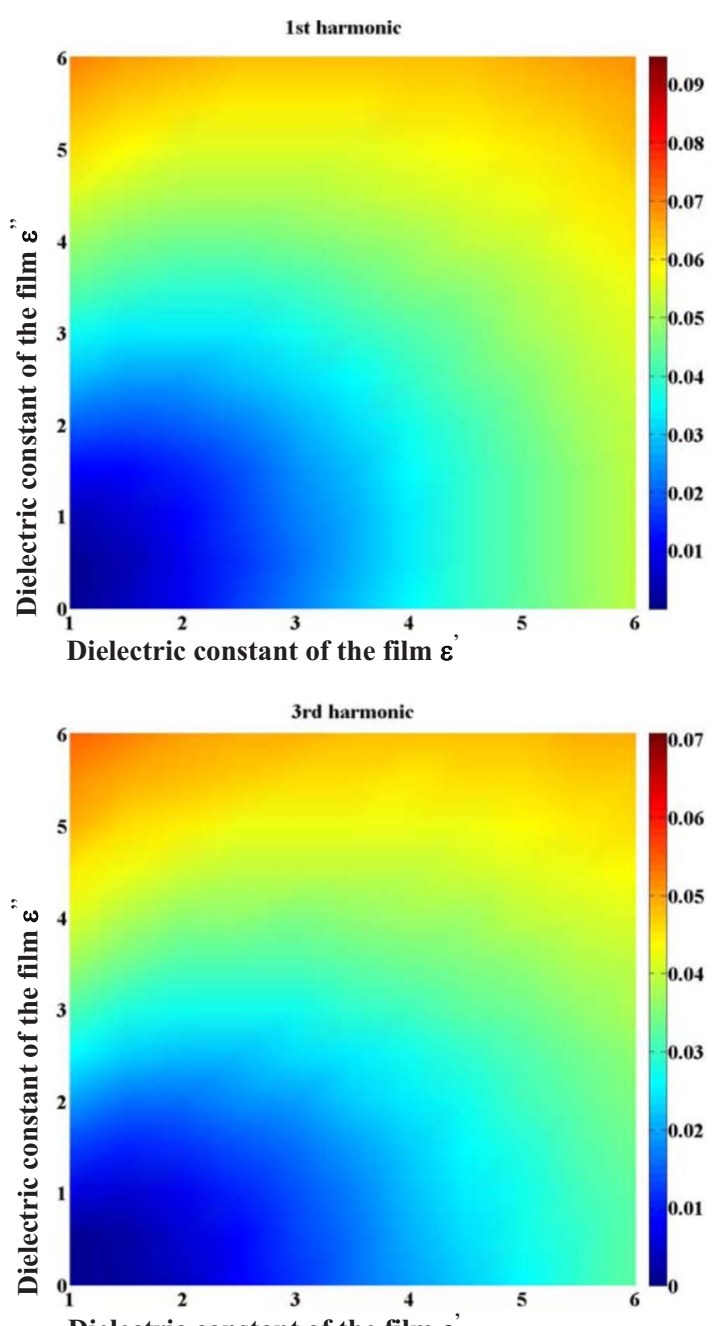

Dielectric constant of the film $\varepsilon$
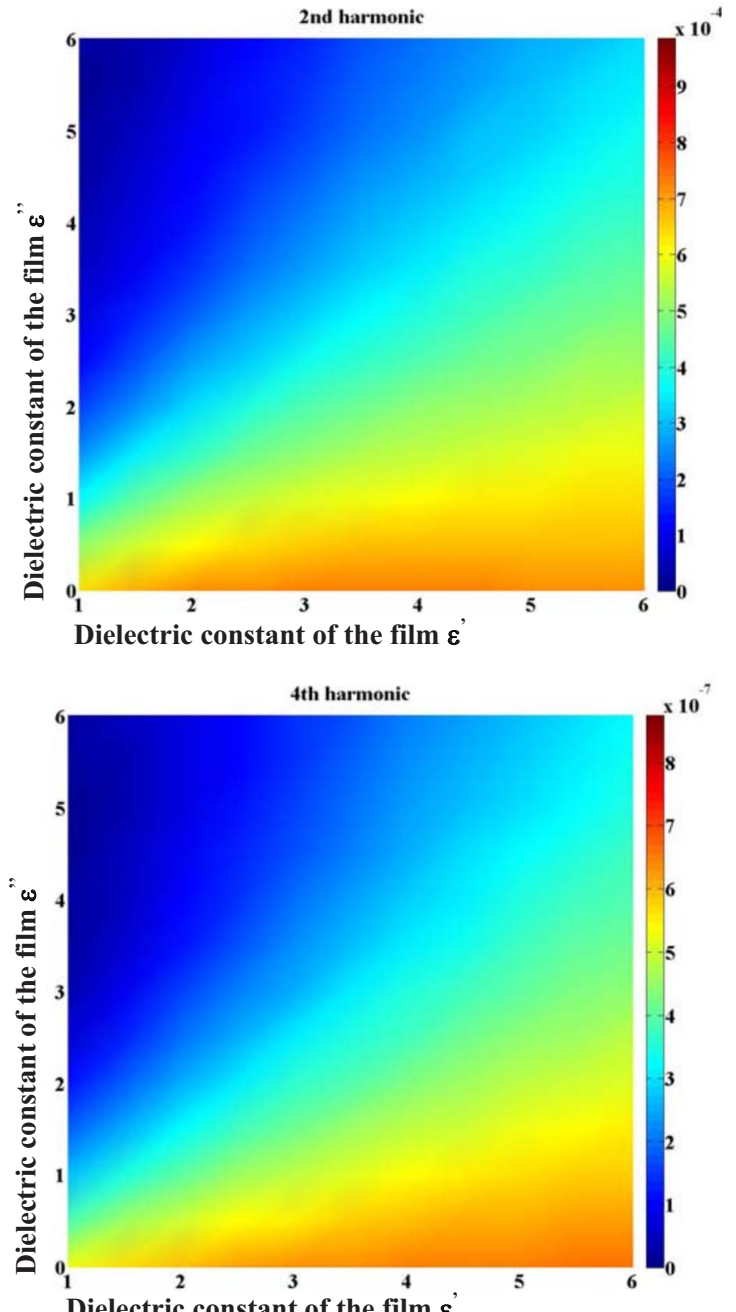

Dielectric constant of the film $\varepsilon$

FIG. 6. (Color online) Intensity maps of the s-NSOM contrast for the HOPG/porphyrin structure on the HOPG system illuminated with $s$-polarized light. Contrast changes are displayed as a function of harmonic number (1-4).

should become darker when the dielectric value of the film material increases. Typically, the near-field signals in s-NSOM increase with increasing dielectric value of the sample (i.e., materials with higher dielectric values appear brighter $\left.{ }^{33}\right)$, also, Aizpurua et al. ${ }^{34}$ show that the near-field signal of a layered system increases, when the dielectric value of the film increases. Our system is significantly different from the systems used in previous experiments, because the substrate with high losses $\left(\operatorname{Im}\left(\varepsilon_{\mathrm{HOPG}}\right)=10\right)$ is used. Previously materials such as $\mathrm{Au}$, mica, $\mathrm{SiO}_{2}, \mathrm{Si}$ with much smaller imaginary part of dielectric constant were used as substrates for s-NSOM studies. When the difference in s-NSOM contrast between porphyrin/HOPG and HOPG are calculated, the fact that $\operatorname{Im}\left(\varepsilon_{\text {porphyrin/HOPG }}\right)$ is smaller than $\operatorname{Im}\left(\varepsilon_{\mathrm{HOPG}}\right)$ needs to be taken into account. Near zero the intensity of scattered s-NSOM amplitude as a function of a real part of optical constant of the substrate decreases. Then it goes through the minimum as a real part of optical constant of the substrate increases. Smaller imaginary part of the optical constant of the substrate corresponds to the deeper minimum on the intensity of scattered s-NSOM amplitude curve (see inset of Fig. 3 from Ref. [33] for illustration). Thus, for our system (porphyrin/HOPG and HOPG) contrast should increase up to some point as real part of the dielectric constant of the film increase. This behavior is presented in Fig. 5.

In order to determine the sensitivity of s-NSOM to the imaginary part of the dielectric constant for the film, numerical modeling of the s-NSOM contrast was carried out for films having a real part of the dielectric constant $[\operatorname{Re}(\varepsilon)]$ in the 1-6 range and an imaginary part $[\operatorname{Im}(\varepsilon)]$ that spanned 0-6 (see Fig. 6) for the $s$-polarized incident light at all harmonics. The oscillatory nature of the solutions is evident from Figs. 6(a)-6(d). Note that these calculations represent the behavior in the absence of a strong absorption process. Changing the wavelength and the angle of the incident light will result in drastic increases in contrast when the wavelength of incident light is the same magnitude as the plasmon resonance of tip-surface junction, or corresponds to a molecular linear absorptive transition, which thereby changes the ratio of the $s$ - and $p$-polarizations for the reflected light. However, even under the mild dielectric variations considered here, the contrast changes by as much as a factor of 5 times over the above-noted range. The magnitude of this contrast variation with dielectric properties indicates the de- 
gree to which local variations in dielectric constant are resolvable.

\section{CONCLUSION}

In summary, we have used an idealized system of an atomically flat metallic surface (HOPG) and an organic monolayer composed of porphyrin, to determine whether the dielectric function and associated properties of thin films can be accessed with s-NSOM. The theoretical analysis and numerical modeling, as well as experimental data, demonstrate that higher harmonic scattering can be used to extract the dielectric properties of materials with tens of nanometers spatial resolution. The third harmonic provides the best lateral resolution $(\sim 50 \mathrm{~nm})$ and dielectric constant contrast for porphyrin film on HOPG.

\section{ACKNOWLEDGMENTS}

The authors gratefully acknowledge financial support from the Nano/Bio Interface Center at the University of Pennsylvania (Grant No. DMR04-25780) and MRSEC (Grant No. DMR05-20020), each of which are funded by the National Science Foundation, and Grant No. SFB 287, funded by the German Science Foundation.

\section{APPENDIX: DERIVATION OF THE SCATTERED LIGHT INTENSITIES FOR $s$-POLARIZED AND p-POLARIZED LIGHT}

The problem of light scattering from a dipole located over a layered media was solved by Chew. ${ }^{35}$ However, only the integral solutions, the so-called Sommerfeld integrals, are known [Eq. (A1) for electric field in the case of $s$-polarized light and Eq. (A2) for electric field in the case of $p$-polarized light].
TABLE I. Dielectric constants for the materials of interest for $\lambda$ $=632.8 \mathrm{~nm}$.

\begin{tabular}{lcc}
\hline \hline Material & Dielectric constant & Ref. \\
\hline Pt/Ir & $-12+19 \times i$ & "Optical constants of solids" by Palik \\
HOPG & $5+10 \times i$ & "Optical constants of solids" by Palik \\
Porphyrin & $3+0 \times i$ & Viseu et al. \\
\hline \hline
\end{tabular}

${ }^{\mathrm{a}}$ Reference 32 .

${ }^{\mathrm{b}}$ Reference 37.

$$
\begin{aligned}
E_{3 L S}= & \frac{i P}{8 \pi \omega \varepsilon_{1}} \cos \varphi \int_{-\infty}^{\infty} d k_{\rho} k_{\rho}^{2} H_{0}^{(1)}\left(k_{\rho} \rho\right)\left[e^{i k_{\rho}|z|}\right. \\
& \left.-R_{12} e^{i k_{1 z}\left(z+2 d_{1}\right)}-T_{12} R_{23} T_{21} e^{i\left[k_{1 z}\left(z+2 d_{1}\right)+k_{2 z}\left(d_{2}-d_{1}\right)\right]}\right],
\end{aligned}
$$

$$
\begin{aligned}
E_{3 V S}= & -\frac{P}{8 \pi \omega \varepsilon_{1}} \cos \varphi \int_{-\infty}^{\infty} d k_{\rho} \frac{k_{\rho}^{3}}{k_{1 z}} H_{0}^{(1)}\left(k_{\rho} \rho\right)\left[e^{i k_{\rho}|z|}\right. \\
& \left.+R_{12} e^{i k_{1 z}\left(z+2 d_{1}\right)}+T_{12} R_{23} T_{21} e^{i\left[k_{1 z}\left(z+2 d_{1}\right)+k_{2 z}\left(d_{2}-d_{1}\right)\right]}\right],
\end{aligned}
$$

where $P$ is the electrical dipole; $\omega$ is the radian frequency of incident light wave; $\varphi$ is the incident angle of light; $d_{1}, d_{2}$, and $z$-see Fig. 2; $R_{x y}$ and $T_{x y}$ are Fresnel coefficients as defined in Ref. 36; $k_{\rho}=k_{1} \rho /\left[\rho^{2}+\left(z+2 d_{1}\right)^{2}\right]^{1 / 2} ; \rho$ is the distance between the tip and the observation point; $H_{0}^{(1)}\left(k_{\rho} \rho\right)$ $\sim \sqrt{\left(2 / \pi k_{\rho} \rho\right)} e^{i k_{\rho} \rho-i \pi / 4}, \rho \rightarrow \infty$, etc.

It is difficult to work with solutions in the form of Sommerfeld integrals, because they cannot be evaluated analytically and are highly oscillatory (the oscillatory nature of the integrals significantly slows convergence). In order to get solutions in a useful form, asymptotic solutions are obtained here using the stationary phase method [Eq. (A3) for the electric field in the case of $s$-polarized light and Eq. (A4) for the electric field in the case of $p$-polarized light)].

$$
\begin{aligned}
E_{3 L S}= & \frac{2}{\rho} k_{\rho}^{3 / 2}\left(f^{\prime \prime}\right)^{-1 / 2} e^{i\left[k_{\rho} \rho+k_{1 z} z\right]}\left[1+\frac{\operatorname{Re}\left[\varepsilon_{1} \cos \alpha_{1}-\sqrt{\varepsilon_{2}^{2}-\varepsilon_{1}^{2} \sin ^{2} \alpha_{1}}\right]}{\operatorname{Re}\left[\varepsilon_{1} \cos \alpha_{1}+\sqrt{\varepsilon_{2}^{2}-\varepsilon_{1}^{2} \sin ^{2} \alpha_{1}} e^{i k_{12} 2 d_{1}}+\right]}\right. \\
& +\frac{\operatorname{Re}\left[2 \varepsilon_{1} \cos \alpha_{1}\right]}{\operatorname{Re}\left[\varepsilon_{1} \cos \alpha_{1}+\sqrt{\varepsilon_{2}^{2}-\varepsilon_{1}^{2} \sin ^{2} \alpha_{1}}\right]} \frac{\operatorname{Re}\left[\varepsilon_{2} \sqrt{1-\left(\frac{\operatorname{Re}\left[\varepsilon_{1}\right]}{\operatorname{Re}\left[\varepsilon_{2}\right]}\right)^{2} \sin ^{2} \alpha_{1}}-\sqrt{\left.\varepsilon_{3}^{2}-\varepsilon_{2}^{2}\left(\frac{\operatorname{Re}\left[\varepsilon_{1}\right]}{\operatorname{Re}\left[\varepsilon_{2}\right]}\right)^{2} \sin ^{2} \alpha_{1}\right]}\right.}{\operatorname{Re}\left[\varepsilon_{2}\left(\frac{\operatorname{Re}\left[\varepsilon_{1}\right]}{\operatorname{Re}\left[\varepsilon_{2}\right]}\right)^{2} \sin ^{2} \alpha_{1}+\sqrt{\varepsilon_{3}^{2}-\varepsilon_{2}^{2}\left(\frac{\operatorname{Re}\left[\varepsilon_{1}\right]}{\operatorname{Re}\left[\varepsilon_{2}\right]}\right)^{2} \sin ^{2} \alpha_{1}}\right]} \\
& \times \frac{\operatorname{Re}\left[2 \varepsilon_{1} \sqrt{\left.1-\left(\frac{\operatorname{Re}\left[\varepsilon_{1}\right]}{\operatorname{Re}\left[\varepsilon_{2}\right]}\right)^{2} \sin ^{2} \alpha_{1}\right]}\right.}{\operatorname{Re}\left[\varepsilon_{2} \sqrt{1-\left(\frac{\operatorname{Re}\left[\varepsilon_{1}\right]}{\operatorname{Re}\left[\varepsilon_{2}\right]}\right)^{2} \sin ^{2} \alpha_{1}}+\sqrt{\varepsilon_{1}^{2}-\varepsilon_{2}^{2}\left(\frac{\operatorname{Re}\left[\varepsilon_{1}\right]}{\operatorname{Re}\left[\varepsilon_{2}\right]}\right)^{2} \sin ^{2} \alpha_{1}}\right]} e^{i\left[k_{12} 2 d_{1}+k_{2 z}\left(d_{2}-d_{1}\right)\right]},
\end{aligned}
$$




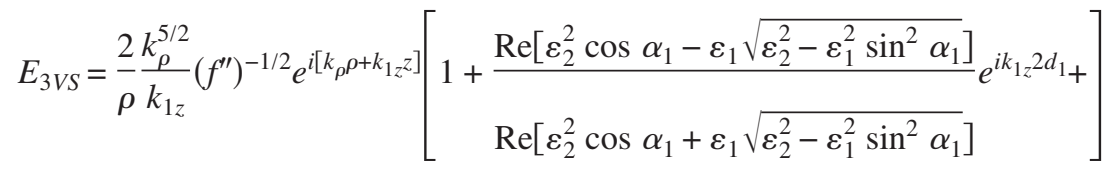

$$
\begin{aligned}
& +\frac{\operatorname{Re}\left[2 \varepsilon_{1} \varepsilon_{2} \cos \alpha_{1}\right]}{\operatorname{Re}\left[\varepsilon_{2}^{2} \cos \alpha_{1}+\varepsilon_{1} \sqrt{\varepsilon_{2}^{2}-\varepsilon_{1}^{2} \sin ^{2} \alpha_{1}}\right]} \frac{\operatorname{Re}\left[\varepsilon_{3}^{2} \sqrt{1-\left(\frac{\operatorname{Re}\left[\varepsilon_{1}\right]}{\operatorname{Re}\left[\varepsilon_{2}\right]}\right)^{2} \sin ^{2} \alpha_{1}-\varepsilon_{2}} \sqrt{\varepsilon_{3}^{2}-\varepsilon_{2}^{2}\left(\frac{\operatorname{Re}\left[\varepsilon_{1}\right]}{\operatorname{Re}\left[\varepsilon_{2}\right]}\right)^{2} \sin ^{2} \alpha_{1}}\right]}{\left.\varepsilon_{3}^{2} \sqrt{1-\left(\frac{\operatorname{Re}\left[\varepsilon_{1}\right]}{\operatorname{Re}\left[\varepsilon_{2}\right]}\right)^{2} \sin ^{2} \alpha_{1}}+\varepsilon_{2} \sqrt{\varepsilon_{3}^{2}-\varepsilon_{2}^{2}\left(\frac{\operatorname{Re}\left[\varepsilon_{1}\right]}{\operatorname{Re}\left[\varepsilon_{2}\right]}\right)^{2} \sin ^{2} \alpha_{1}}\right]} \\
& \times \frac{\operatorname{Re}\left[2 \varepsilon_{2} \varepsilon_{1} \sqrt{1-\left(\frac{\operatorname{Re}\left[\varepsilon_{1}\right]}{\operatorname{Re}\left[\varepsilon_{2}\right]}\right)^{2} \sin ^{2} \alpha_{1}}\right]}{\operatorname{Re}\left[\varepsilon_{1}^{2} \sqrt{1-\left(\frac{\operatorname{Re}\left[\varepsilon_{1}\right]}{\operatorname{Re}\left[\varepsilon_{2}\right]}\right)^{2} \sin ^{2} \alpha_{1}}+\varepsilon_{2} \sqrt{\varepsilon_{1}^{2}-\varepsilon_{2}^{2}\left(\frac{\operatorname{Re}\left[\varepsilon_{1}\right]}{\operatorname{Re}\left[\varepsilon_{2}\right]}\right)^{2} \sin ^{2} \alpha_{1}}\right]} e^{i\left[k_{1 z^{2}} 2 d_{1}+k_{2 z}\left(d_{2}-d_{1}\right)\right]} .
\end{aligned}
$$

The time dependence of the electric fields was evaluated within the MATLAB environment. Fast Fourier transforms of the time series were used to determine the intensity of each harmonic. For the numerical modeling the following parameters were used: $P=1$ is the dipole induced by incident light in a tip (the exact value does not matter, because we are calculating s-NSOM contrast and these parameters cancel), the incident angle $=65^{\circ}, \omega=2 \pi c / 6328 \AA^{-1}, \varepsilon_{1}=1$ is the dielectric constant of vacuum, $\varepsilon_{2}$ (dielectric constant of the film) is the variable, $\varepsilon_{3}=5+10 i$ (dielectric constant of graphite for the light with wavelength of $632.8 \mathrm{~nm}$ ), the tip oscillates near the surface with amplitude $20 \AA$, the closest distance between the tip and surface is $1 \AA$, and the frequency of the tip oscillations is $150 \mathrm{kHz}$ (Table I). The film thickness is $15 \AA$.

${ }^{1}$ M. Moreno-Moreno, A. Raman, J. Gomez-Herrero, and R. Reifenberger, Appl. Phys. Lett. 88, 193108 (2006).

${ }^{2}$ S. Crittenden, A. Raman, and R. Reifenberger, Phys. Rev. B 72, 235422 (2005).

${ }^{3}$ S. V. Kalinin and D. A. Bonnell, Appl. Phys. Lett. 78, 1306 (2001).

${ }^{4}$ E. Bussmann and C. C. Williams, Rev. Sci. Instrum. 75, 422 (2004).

${ }^{5}$ U. Zerweck, C. Loppacher, T. Otto, S. Grafstrom, and L. M. Eng, Phys. Rev. B 71, 125424 (2005).

${ }^{6}$ Science of Microscopy, edited by P. W. Hawkes and J. C. H. Spence (Springer, New York, 2006), p. 1400.

${ }^{7}$ S. C. Kehr, M. Cebula, O. Mieth, T. Hartling, J. Seidel, S. Grafstrom, L. M. Eng, S. Winnerl, D. Stehr, and M. Helm, Phys. Rev. Lett. 100, 256403 (2008).

${ }^{8}$ S. Grafstrom, J. Appl. Phys. 91, 1717 (2002).

${ }^{9}$ R. Hillenbrand, T. Taubner, and F. Keilmann, Nature (London) 418, 159 (2002).

${ }^{10}$ F. Zenhausern, M. P. Oboyle, and H. K. Wickramasinghe, Appl. Phys. Lett. 65, 1623 (1994).

${ }^{11}$ T. Taubner, R. Hillenbrand, and F. Keilmann, J. Microsc. 210, 311 (2003).

${ }^{12}$ R. Hillenbrand and F. Keilmann, Appl. Phys. Lett. 80, 25 (2002).
${ }^{13}$ R. Hillenbrand, B. Knoll, and F. Keilmann, J. Microsc. 202, 77 (2001).

${ }^{14}$ R. Hillenbrand and F. Keilmann, Phys. Rev. Lett. 85, 3029 (2000).

${ }^{15}$ B. Knoll and F. Keilmann, Opt. Commun. 182, 321 (2000).

${ }^{16}$ G. Wollny, E. Brundermann, Z. Arsov, L. Quaroni, and M. Havenith, Opt. Express 16, 7453 (2008).

${ }^{17}$ R. Garcia and R. Perez, Surf. Sci. Rep. 47, 197 (2002).

${ }^{18}$ S. Schneider, J. Seidel, S. Grafstrom, L. M. Eng, S. Winnerl, D. Stehr, and M. Helm, Appl. Phys. Lett. 90, 143101 (2007).

${ }^{19}$ S. C. Schneider, S. Grafstrom, and L. M. Eng, Phys. Rev. B 71, 115418 (2005).

${ }^{20}$ R. Hillenbrand and F. Keilmann, Appl. Phys. B 73, 239 (2001).

${ }^{21}$ M. Brehm, H. G. Frey, R. Guckenberger, R. Hillenbrand, D. Kazantsev, F. Keilmann, N. Ocelic, and T. Taubner, J. Korean Phys. Soc. 47, S80 (2005).

${ }^{22}$ F. Keilmann and R. Hillenbrand, Philos. Trans. R. Soc. London, Ser. A 362, 787 (2004).

${ }^{23}$ H. T. Uyeda, Y. X. Zhao, K. Wostyn, I. Asselberghs, K. Clays, A. Persoons, and M. J. Therien, J. Am. Chem. Soc. 124, 13806 (2002).

${ }^{24}$ M. P. Nikiforov, U. Zerweck, P. Milde, C. Loppacher, T. H. Park, H. T. Uyeda, M. J. Therien, L. Eng, and D. Bonnell, Nano Lett. 8, 110 (2008).

${ }^{25}$ J. M. Vigoureux, C. Girard, and D. Courjon, Opt. Lett. 14, 1039 (1989).

${ }^{26}$ D. Vanlabeke and D. Barchiesi, J. Opt. Soc. Am. A Opt. Image Sci. Vis 9, 732 (1992).

${ }^{27}$ S. V. Sukhov, Opt. Spectrosc. 98, 301 (2005).

${ }^{28}$ W. Denk and D. W. Pohl, J. Vac. Sci. Technol. B 9, 510 (1991).

${ }^{29}$ D. Van Labeke and D. Barchiesi, J. Opt. Soc. Am. A Opt. Image Sci. Vis 10, 2193 (1993).

${ }^{30}$ D. Barchiesi and D. Van Labeke, J. Mod. Opt. 40, 1239 (1993).

${ }^{31}$ M. F. Xiao and S. Bozhevolnyi, Opt. Commun. 130, 337 (1996).

${ }^{32}$ E. D. Palik, Handbook of Optical Constants of Solids (Elsevier, New York, 1991).

${ }^{33}$ M. B. Raschke and C. Lienau, Appl. Phys. Lett. 83, 5089 (2003).

${ }^{34}$ J. Aizpurua, T. Taubner, F. J. de Abajo, M. Brehm, and R. Hillenbrand, Opt. Express 16, 1529 (2008).

${ }^{35}$ W. C. Chew, Waves and Fields in Inhomogeneous Media (IEEE, New York, 1994).

${ }^{36}$ J. D. Jackson, Classical Electrodynamics (Wiley, New York, 1999).

${ }^{37}$ T. M. R. Viseu, G. Hungerford, and M. I. C. Ferreira, J. Phys. Chem. B 106, 1853 (2002). 